\title{
BODY MASSES AND MEASUREMENTS OF BIRDS FROM SOUTHERN ATLANTIC FOREST, BRAZIL ${ }^{1}$
}

\author{
Bianca L. Reinert ${ }^{2}$ \\ Júlio C. Pinto ${ }^{3}$ \\ Marcos R. Bornschein ${ }^{2}$ \\ Mauro Pichorim 4 \\ Miguel Â. Marini ${ }^{5}$
}

\begin{abstract}
Five hundred and eigh body masses of 74 forest birds, and measurements of wing, tail, tarsus and beak of 14 poorly known species mist-netted at two sites in the Atlantic Forest of eastern Paraná State, southern Brazil, are presented.

KEY WORDS. Birds, body masses, measurements, Atlantic Forest
\end{abstract}

The Atlantic Forest is one of the world hotspots of biodiversity (MYERS 1988 ) with high levels of endemism (HAFFER 1974; CRACRAFT 1985; SCOTT \& BROOKE 1985), but also with high levels of deforestation (OEDEKOVEN 1980; REDFORD 1989). In spite of the importance of this ecosystem, taxonomic and basic biological data (see e.g. DAVIS 1946; SCOTT \& BROOKE 1985; TEIXEIRA et al. 1986, 1993; WILlis 1979, 1989) are relatively scarce for most birds of the region. Examples of taxonomic revisions of birds present on the sampled region include the reports by SILVA \& STOTZ (1992) for the now politypic Heliobletus contaminatus Berlepsch, 1885, by RAPOSO \& TEIXEIRA (1992) and WiLlis (1992) for Chamaeza meruloides Vigors, 1825, misidentified as C. campanisoma (Lichtenstein, 1818), and by WILLIS \& ONIKI (1992) for Phylloscartes kronei Willis \& Oniki, 1992, a new species misidentified as $P$. ventralis (Temminck, 1824). Other example of the little knowledge about Atlantic Forest birds is the recent discovery of Stymphalornis acutirostris Bornschein, Reinert \& Teixeira, 1995, a new genus and species of Formicariidae from the Paraná coast (BoRNSCHEIN et al. 1995). Previous studies that reported body masses of birds that occur in the Atlantic Forest region include BELton (1984), TEIXEIRA et al. (1986, 1987, 1988, 19891993), STORER (1989), and DUNNING (1992), which compiled body masses of birds of the world.

This paper intends to improve the knowledge about body masses of birds from southern Atlantic Forest in Paraná State, and provide measurements of some poorly known species, mostly endemic to this region.

1) Contribuição número 979 do Departamento de Zoologia, Universidade Federal do Paraná.

2) Divisão de Museu de História Natural Capão da Imbuia. Rua Prof. Benedito Conceição 407, 82810-080 Curitiba, Paraná, Brasil.

3) Rua Airton Plaisant 1959, bloco 3, ap. 102, 80330-010 Curitiba, Paraná, Brasil.

4) Departamento de Zoologia, Universidade Federal do Paraná. Caixa Postal 19020, 81531-990 Curitiba, Paraná, Brasil.

5) Departamento de Biologia Geral, Universidade Federal de Minas Gerais. 30161-970 Belo Horizonte, Minas Gerais, Brasil. 


\section{MATERIAL AND METHODS}

Birds were captured with mist-nets in July-August 1991, March 1992, February 1993 and July-August 1995, at two sites in Paraná State: Mananciais da Serra ( $25^{\circ} 30^{\prime} \mathrm{S}, 48^{\circ} 57^{\prime} \mathrm{W}$; c. $1.050-1.150 \mathrm{~m}$ a.s.l.), municipality of Piraquara (all

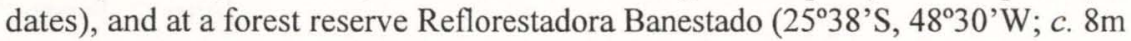
a.s.l.), municipality of Paranaguá (in July 1991 and August 1995). In Mananciais da Serra it was sampled patches of primary and secondary dense montane forest, and in Reflorestadora Banestado a palm-heart (Euterpe edulis, Arecaceae) managed forest, classified respectively as "Floresta Ombrófila Densa Montana" and "Floresta Ombrófila Densa das Terras Baixas" (sensu Veloso et al. 1991).

Body masses were taken from live mist-netted birds with 30,50 , and $100 \mathrm{~g}$ Pesola spring scales, and recorded to the nearest $0.5 \mathrm{~g}$. Birds were captured mostly in the morning (6:00-14:00). Sample sizes for each particular mass value are given in parenthesis. For samples larger than 10 was given mean more or less standard deviation, range and sample in parenthesis. Sex identification $(\mathrm{M}=$ male, $\mathrm{MJ}=$ juvenile male, $\mathrm{F}=$ female, $\mathrm{I}$ = indeterminate, $\mathrm{IJ}=$ indeterminate juvenile) was based on plumage characteristics. Body measurements were taken with a metal Caliper to the nearest $0.1 \mathrm{~mm}$ by only one of the authors. It was taken the chord (non-flattened wing), the tail (measured from the uropygial gland to the tip of the tail), standard tarsus-metatarsus length and nostril (anterior end of the nostril to the tip of the beak) measurements. Taxonomy of species follows SIBLEY \& MONROE (1990).

Table I. Measurements in milimeters of four body parts of some poorly known birds, mostly endemic to Atlantic Forest.

\begin{tabular}{|c|c|c|c|c|}
\hline \multirow{2}{*}{ Species } & \multicolumn{4}{|c|}{ Measurements ${ }^{1}$ (meam \pm 1 standard deviation, range) ${ }^{2}$} \\
\hline & Wing & Tail & Tarsus & Beak \\
\hline Platyrinchus leucoryphus $(n=1)$ & 69.9 & 48.0 & 15.0 & 8.6 \\
\hline Carpornis cucullatus $(n=4)$ & $\begin{array}{c}112.3 \pm 2.9 \\
(109.6-113.3)\end{array}$ & $\begin{array}{c}108.1 \pm 7.7 \\
(102.1-118.7)\end{array}$ & $\begin{array}{c}24.2 \pm 0.6 \\
(23.9-25.1)\end{array}$ & $\begin{array}{c}9.2 \pm 0.7 \\
(8.6-10.1)\end{array}$ \\
\hline Mymotherula gularis $(n=1)$ & 49.7 & 32.0 & 22.3 & 7.6 \\
\hline Drymophila malura $(n=4)$ & $\begin{array}{c}54.2 \pm 1.1 \\
(53.2-55.6)\end{array}$ & $\begin{array}{c}83.1 \pm 2.0 \\
(80.5-84.8)\end{array}$ & $\begin{array}{c}23.4 \pm 0.3 \\
(23.1-23.7)\end{array}$ & $\begin{array}{c}8.0 \pm 0.3 \\
(7.7-8.3)\end{array}$ \\
\hline Syndactyla rufosuperciliata $(n=9)$ & $\begin{array}{c}70.5 \pm 2.0 \\
(67.5-72.9)\end{array}$ & $\begin{array}{c}82.7 \pm 4.2 \\
(76.0-90.0)\end{array}$ & $\begin{array}{c}23.8 \pm 0.7 \\
(22.6-24.9)\end{array}$ & $\begin{array}{c}12.4 \pm 0.7 \\
(11.5-13.9)\end{array}$ \\
\hline Philydor amaurotis $(n=24)^{3}$ & $\begin{array}{c}70.1 \pm 3.4 \\
(65.6-77.6)\end{array}$ & $\begin{array}{c}76.5 \pm 4.0 \\
(65.7-82.6)\end{array}$ & $\begin{array}{c}20.2 \pm 0.6 \\
(19.1-21.1)\end{array}$ & $\begin{array}{c}10.6 \pm 0.4 \\
(9.8-11.5)\end{array}$ \\
\hline Sclerurus scansor $(n=1)$ & 84.1 & 74.0 & 24.8 & 17.2 \\
\hline Heliobletus contaminatus $(n=2)$ & $60.9,63.0$ & $54.9,56.5$ & $18.5,19.4$ & $9.3,9.4$ \\
\hline Campylorhamphus falcularis $(n=2)$ & $96.8,104.4$ & $107.2,108.9$ & $22.9,23.3$ & 64.6. 70.6 \\
\hline Grallaria varia $(n=1)$ & 125.3 & 57.2 & 61.8 & 18.8 \\
\hline Thraupis cyanoptera $(n=1)$ & 94.5 & 78.4 & 21.9 & 10.3 \\
\hline Tangara desmaresti $(n=3)$ & $\begin{array}{c}67.9 \pm 2.3 \\
(66.2-70.6)\end{array}$ & $\begin{array}{c}63.2 \pm 1.7 \\
(62.0-65.1)\end{array}$ & $\begin{array}{c}20.1 \pm 0.6 \\
(19.6-20.8)\end{array}$ & $\begin{array}{c}7.2 \pm 0.1 \\
(7.2-7.3)\end{array}$ \\
\hline Haplospiza unicolor $(n=11)$ & $\begin{array}{c}60.9 \pm 1.7 \\
(58.1-62.9)\end{array}$ & $\begin{array}{c}55.8 \pm 1.8 \\
(54.1-21.0)\end{array}$ & $\begin{array}{c}19.5 \pm 0.8 \\
(18.5-21.0)\end{array}$ & $\begin{array}{c}8.0 \pm 0.3 \\
(7.6-8.3)\end{array}$ \\
\hline Amaurospiza moesta $(n=3)$ & $\begin{array}{c}59.6 \pm 2.0 \\
(58.0-61.8)\end{array}$ & $\begin{array}{c}61.7 \pm 0.2 \\
(61.5-61.9)\end{array}$ & $\begin{array}{c}20.2 \pm 0.1 \\
(20.1-20.2)\end{array}$ & $\begin{array}{c}7.1 \pm 0.4 \\
(6.8-7.6)\end{array}$ \\
\hline
\end{tabular}

1) Measurements as explained in the text; 2 ) For samples of one or two was given exact values for each individual; 3) Sample size of 23 for tail. 


\section{RESULTS}

Listed bellow are 508 body masses of 74 forest birds, and in the table I the measurements of wing, tail, tarsus and beak of 14 poorly known species.

Picumnus temminckii Lafresnaye, 1845: M 10.5; F 11.5

Veniliornis spilogaster (Wagler, 1827): M 40.0; F 40.5

Trogon rufus (Gmelin, 1788): F 62.0

Phaethornis eurynome (Lesson, 1832): I $5.2 \pm 0.5(\mathrm{~N}=15)$

Phaethornis squalidus (Temminck, 1822): I 3.0, 3.5 (2)

Ramphodon naevius (Dumont, 1818): M 9.0: F 6.0, 6.5; I 7.0

Thalurania glaucopis (Gmelin, 1788): M 5.0 (2), 5.5 (4); F 4.0 (2), 4.5 (2); I 4.0 (1), 4.5 (2), 5.0 (3)

Amazilia versicolor (Vieillot, 1818): $\mathrm{F} 4.5$

Clytolaema rubricauda (Boddaert, 1783): M 8.0, 8.5, 9.0, 9.5, 10.0; F 6.5 (2), 7.0 (2), 7.5 (4); I 6.5, 7.0, 7.5

Geotrygon montana (Linnaeus, 1758): IJ 88.5

Mionectes rufiventris Cabanis, 1846: I 13.0 (2), 14.0, 14.5, 15.5

Leptopogon amaurocephalus Tschudi, 1846: I 12.0, 12.5 (2)

Hemitriccus obsoletus (Miranda Ribeiro, 1906): I 11.6 $1.0(\mathrm{~N}=24)$

Todirostrum plumbeiceps Lafresnaye, 1846: I 5.5 (2), 6.0 (2)

Phylloscartes difficilis (Ihering \& Ihering, 1907): I 6.5, 7.0 (2)

Phylloscartes ventralis: I 7.5, 8.0, 8.5

Tolmomyias sulphurescens (Spix, 1825): I 17.0, 17.5 (3), 18.0

Platyrinchus mystaceus Vieillot, 1818: M 9.4 $\pm 0.9(\mathrm{~N}=12)$; F 7.5 (3), 8.5, 10.0; I 9.5

Platyrinchus leucoryphus Wied, 1831: I 17.0

Onychorhynchus coronatus (Müller, 1776): I 23.5

Myiobius barbatus (Gmelin, 1789): I 11.0, 12.0 (2), 12.4

Lathrotriccus euteri (Cabanis, 1868): I 10.0, 10.5, 11.0 (2), 12.0 (3), 13.0

Attila phoenicurus Pelzeln, 1868: I $32.3 \pm 1.9(\mathrm{~N}=11)$

Attila rufus (Viellot, 1819): I 38.0, 42.0

Schiffornis virescens (Lafresnaye, 1838): I 23.0, 24.0, 25.0, 26.0 (4), 26.5, 27.0; M 26.0

Carpornis cucullatus (Swainson, 1821): M 72.0, 74.0 (2), 75.0; F 66.5; I 69.0

Chiroxiphia caudata (Shaw \& Nodder, 1793): M 26.0 (6), 28.0 (2), 29.5; MJ 24.5;

F 23.5 (2), 24.0, 25.5, 37.0; I 23.0, 23.5(2), 25.0, 25.5, 27.0, 28.5, 33.0

Thamnophilus caerulescens Vieillot, 1816: M 23.5; F 21.0 (2)

Dysithamnus mentalis (Temminck, 1823): F 11.0, 12.0; M 11.5, 12.0 (2); I 12.5

Myrmotherula gularis (Spix, 1825): M 11.0 (4), 13.0; F 11.5

Myrmotherula unicolor Ménétriès, 1835: F 11.5

Drymophila malura (Temminck,1825): M 12.0, 12.5, 13.0 (2), 13.5 (2), 16.5; MJ

$12.5 ; \mathrm{F} 11.5,12.5 ; \mathrm{I} 11.5$

Pyriglena leucoptera (Vieillot, 1818): F 26.0

Synallaxis ruficapilla Vieillot, 1819: I $13.8 \pm 0.7(\mathrm{~N}=18)$

Clibanornis dendrocolaptoides (Pelzeln, 1859): I 42.0 
Lochmias nematura (Lichtenstein, 1823): I 22.0, 23.0

Syndactyla rufosuperciliata (Lafresnaye, 1832): I 25.6 $\pm 1.4(\mathrm{~N}=12)$

Philydor amaurotis (Temminck, 1823): I 19.2 $\pm 1.2(\mathrm{~N}=29)$

Philydor rufus (Vieillot, 1818): I 22.0 (2), 23.0 (2), 24.0

Automolus leucophthalmus (Wied, 1821): I 33.0, 36.5, 38.0

Sclerurus scansor (Ménétriès, 1835): I 39.0, 41.0

Heliobletus contaminatus: I 12.5, 13.0, 14.0 (2), 15.5

Xenops minutus (Sparrman, 1788): I 8.5 (3)

Dendrocincla fuliginosa (Vieillot, 1818): I 38.5, 41.0, 41.5

Sittasomus griseicapillus (Vieillot, 1818): I 12.4 $\pm 0.8(\mathrm{~N}=13)$

Dendrocolaptes platyrostris Spix, 1825: I 56.0, 57.5, 58.5, 60.0

Lepidocolaptes fuscus (Vieillot, 1818): I 19.4 $\pm 1.2(\mathrm{~N}=16)$

Campylorhamphus falcularius (Vieillot, 1822): I 37.0, 40.5, 41.5

Chamaeza sp. cf. C. campanisoma: I 84.5, 97.0

Chamaeza ruficauda (Cabanis \& Heine, 1859): I 63.5, 76.0

Grallaria varia (Boddaert, 1783): I 134.0

Conopophaga lineata (Wied, 1831): M 21.1 $1.6(\mathrm{~N}=11)$; F 20.5; IJ 20.0 (2); I 19.0, 21.0, 22.0, $23.0(2)$

Cyclarhis gujanensis (Gmelin, 1789): I 29.0, 30.0, 31.0, 31.5; IJ 27.5

Hylophilus poicilotis Temminck, 1822: I 10.0 (2), 12.0

Platycichla flavipes (Vieillot, 1818): M 56.0 (2), 67.0, 77.0; MJ 53.5; I 50.0, 60.0, 61.5

Turdus rufiventris Vieillot, 1818: I 70.0, 73.0, 73.5, 74.0, 80.0

Turdus amaurochalinus Cabanis, 1851: I 59.0

Turdus albicollis Vieillot, 1818: I 68.0 $6.3(\mathrm{~N}=23)$

Basileuterus culicivorus (Lichtenstein, 1830): I 9.7 $\pm 0.9(\mathrm{~N}=14)$

Basileuterus leucoblepharus (Vieillot, 1817): I 15.1 $\pm 0.9(\mathrm{~N}=26)$

Pyrrhocoma ruficeps (Strickland, 1844): M 16.0, 17.0

Tachyphonus coronatus (Vieillot, 1822): M 29.5; F 20.5, 26.0, 26.5

Trichothraupis melanops (Vieillot, 1818): M 24.0; F 23.5, 24.0 (2), 24.5, 25.0, 25.5; I 25.0

Habia rubica (Vieillot, 1817): I 35.0

Thraupis sayaca (Linnaeus, 1766): I 40.5

Thraupis cyanoptera (Vieillot, 1817): I 40.0

Stephanophorus diadematus (Temminck, 1823): I 28.5, 30.5, 31.0, 32.0 (2), 34.0

Euphonia pectoralis (Latham, 1801): M 15.0, 15.5

Tangara desmaresti (Vieillot, 1819): I 19.5, 21.5 (2)

Haplospiza unicolor Cabanis, 1851: M 14.0, 15.0, 15.5, 16.0 (2), 16.5, 17.0, 17.5;

F $14.7 \pm 1.4(\mathrm{~N}=10) ; \mathrm{I} 11.0,14.5(2), 15.5(2), 17.0$

Poospiza lateralis (Nordmann, 1835): I 19.5

Amaurospiza moesta (Hartlaub, 1853): MJ 12.5; M 14.5; F 13.0; I 13.5

Saltator similis Lafresnaye \& d'Orbigny, 1837: I 49.0

Cacicus chrysopterus (Vigors, 1825): I 38.0 


\section{DISCUSSION}

Several Atlantic Forest endemic birds are poorly known either alive or at museum collections. Body masses and measurements provided here represent, thus, one of the few and first reports for these species.

Some species had highly variable body masses (Platycichla flavipes: 50 to $77 \mathrm{~g}$; Chiroxiphia caudata females 23.5 to $37.0 \mathrm{~g}$ ). An analysis of the body masses of the best sampled Atlantic Forest endemic birds shows that males and females had similar values for Haplospiza unicolor, whereas females where lighter in some hummingbirds (e.g. Thalurania glaucopis and Clytolaema rubricauda) and in some passeriforms, such as Carpornis cucullatus, Chiroxiphia caudata and Drymophila malura.

Some of the sampled Furnariidae which occur syntopically in southern Atlantic Forest have beak (see Tab. I) and body masses differences [Syndactyla rufosuperciliata $(\overline{\mathrm{x}}=12.4 \mathrm{~mm}$ and $25.6 \mathrm{~g})$, Philydor amaurotis $(\overline{\mathrm{x}}=10.6 \mathrm{~mm}$ and $19.2 \mathrm{~g})$ and Heliobletus contaminatus $(\mathrm{x}=9.3 \mathrm{~mm}$ and $13.8 \mathrm{~g})]$, which may reflect an avoidance of diet overlap. Other closely related species (e.g. Myrmotherula gularis and M. unicolor; Thraupis sayaca and T. cyanoptera), however, have apparently similar body masses.

These examples are only illustrative of the potential importance of studies of body masses and measurements of birds.

ACKNOWLEDGEMENTS. Field work was partially supported by grants from the Dept. of Ecology, Ethology, and Evolution (University of Illinois), Sigma Xi, Western Bird Banding Association, and Graduate College (Univiversity of Illinois) and a Ph. D. fellowship from Coordenação de Aperfeiçoamento de Pessoal de Nível Superior (CAPES) to M.Â.M, and from the Conselho Nacional de Desenvolvimento Cientifico e Tecnológico (CNPq) to B.L.R., M.R.B. and M.P. We thank Centro de Pesquisas para Conservação das Aves Silvestres (CEMAVE-IBAMA) for providing metal bands and banding authorizations, Companhia de Saneamento do Paraná (Sanepar) and Banco do Estado do Paraná (Banestado) for allowing the study of birds in their properties. Carla S. Coleto, Fernando C. Straube, Márcia C.R. do Valle and Regina Yabe for help during part of the field work.

\section{REFERENCES}

Belton, W. 1984. Birds of Rio Grande do Sul, Brazil. Bull. Amer. Mus. Nat. Hist. 178: $369-631$.

Bornschein, M.R.; B.L. ReIneRT \& D.M. TEIXEIRA. 1995. Um novo Formicariidae do sul do Brasil (Aves, Passeriformes). Série Publicação Técnico-Científica do Instituto Iguaçu de Pesquisa e Preservação Ambiental, Rio de Janeiro, no. 1, 18p.

CRACRAFT, J. 1985. Historical biogeography and patterns of differentiation within the South American avifauna: Areas of endemism. Ornith. Monogr. 36: 49-84.

DAVIS, D.E. 1946. A seasonal analysis of mixed flocks of birds of Brazil. Ecology 27: $168-181$. 
DUNNING JR., J.B. 1992. CRC handbook of avian body masses. Florida, CRC Press, $371 \mathrm{p}$.

Haffer, J. 1974. Avian speciation in tropical South America. Publ. Nuttall. Ornith. Club 14: 179-312.

MYERS, N. 1988. Threatened biotas: "Hotspots" in tropical forests. Environmentalist 8: 1-20.

OEdeKoven, K. 1980. The vanishing forest. Environ. Policy and Law 6: 184-185.

Raposo, M.A. \& D.M. TeIXeIRA. 1992. Revalidação de Chamaeza meruloides Vigors, 1825 (Aves, Formicariidae). Bol. Mus. Nac., N.S. Zool., 350: 1-11.

REDFORD, K.H. 1989. Monte Pascoal - indigenous rights and conservation in conflict. Oryx 23: 33-36.

ScotT, D.A. \& M.L. BRooKe. 1985. The endangered avifauna of southeastern Brazil: a report on the BOU/WWF expeditions of 1980/81 and 1981/82. ICBP Tech. Publ. 4: 115-139.

SiBley, C.G. \& B.L. MONROE JR. 1990. Distribution and taxonomy of birds of the world. New Haven, Yale University Press, 1111 p.

SilvA, J.M.C. \& D. Stotz. 1992. Geographic variation in the Sharp-billed Treehunter Heliobletus contaminatus. Bull. B.O.C. 112: 98-101.

Storer, R.W. 1989. Notes on Paraguayan birds. Occ. Pap. Mus. Zool. Univ. Michigan 719: 1-21.

Veloso, H.P.; A.L.R. Rangel Filho \& J.C.A. Lima. 1991. Classificação da vegetação brasileira adaptada a um sistema universal. Rio de Janeiro, Fundação Instituto Brasileiro de Geografia e Estatística, 123p.

TEIXEIRA, D.M.; J.B. NACINOVIC \& M.S. TAVARES. 1986. Notes on some birds of northeastern Brazil. Bull. B.O.C. 106 (2): 70-74.

TeIXEIRA, D.M.; J.B. NACINOVIC \& F.B. Pontual. 1987. Notes on some birds of northeastern Brazil (2). Bull. B.O.C. 107 (4): 151-157.

TEIXEIRA, D.M.; J.B. NACINOVIC \& G. LUIGI. 1988. Notes on some birds of northeastern Brazil (3). Bull. B.O.C. 108 (2): 75-79.

- 1986-1993. Notes on some birds of northeastern Brazil (4). Bull. B.O.C. 109 (3): 152-157.

Teixeira, D.M.; R. Otoch; G. Luigi; M.A. Raposo \& A.C.C. DE Almeida. 1986-1993. Notes on some birds of northeastern Brazil (5). Bull. B.O.C. 113 (1): 48-52.

WILLIS, E.O. 1979. The composition of avian communities in remanescent woodlots in Southern Brazil. Pap. Avul. Dept. Zool. 33: 1-25.

1989. Mimicry in bird flocks of cloud forests in Southeastern Brazil. Rev. Brasil. Biol. 49: 615-619.

1992. Three Chamaeza antthrushes in eastern Brazil (Formicariidae). Condor 94: 110-116.

Willis, E.O. \& Y. ONIKI. 1992. A new Phylloscartes (Tyrannidae) from southeastern Brazil. Bull. B.O.C. 112 (3): 158-165.

Recebido em 28.III.1996; aceito em 25.XI.1996. 